\title{
Does fine-needle aspiration biopsy still have a place in the diagnosis of breast lesions?
}

\author{
Marzanna Obrzut ${ }^{1}$, Marian Cholewa², Jakub Baran², Agnieszka Obrzut-Palusińska ${ }^{3}$, Ewa Kurczab ${ }^{4}$ \\ Institute of Nursing and Health Sciences, Faculty of Medicine, University of Rzeszow, Poland \\ ${ }^{2}$ Department of Biophysics, Faculty of Mathematics and Natural Sciences, University of Rzeszow, Poland \\ ${ }^{3}$ Medical University of Lodz, Poland \\ ${ }^{4}$ Department of Clinical Pathomorphology, Frederick Chopin Clinical Provincial Hospital No 1 Rzeszow, Poland
}

\begin{abstract}
Introduction: Core needle biopsy is a preferable breast biopsy technique under ultrasound guidance. However, fine-needle biopsy is considered equally popular.

Aim of the study: To redefine the role of fine-needle aspiration biopsy (FNAB) in the diagnosis of breast lesions.

Material and methods: We retrospectively analysed the medical records of 680 patients who underwent breast ultrasound examination. In most cases, no pathologic structures were observed within the mammary glands. In 321 patients, the presence of focal lesions was revealed, and 107 patients in this group qualified for FNAB according to current recommendations. Patients with suspicious smears were referred for core needle or surgical biopsy. Patients with benign cytological smears underwent repeated ultrasound checks at 6-month intervals during the following year.

Results: All the smears were diagnostic. The vast majority of the results were categorised as benign lesions. Cancer cells were detected in six women. In one patient the lesion was classified as suspicious, probably malignant. In all of these cases, open biopsy was performed, and histopathological examination confirmed the presence of a malignant tumour. The patients were given appropriate oncological treatment. For women with benign or suspicious, but probably benign, lesions, breast ultrasound was performed twice at six-month intervals. Control tests showed no significant changes compared to the baseline examination. None of the patients required extensive additional diagnostic tests.
\end{abstract}

Conclusions: FNAB is a reliable method of assessing pathologic lesions in mammary glands.

Key words: breast lesion, ultrasound examination, BI-RADS, fine-needle aspiration biopsy.

\section{Introduction}

Ultrasound examination is a recognised diagnostic method for women with a dense breast structure. Ultrasound diagnosis is inexpensive, does not require the use of potentially harmful X-rays, and facilitates highly accurate differentiation between solid and cystic lesions and assessment of the morphology of lactiferous ducts. On the basis of an ultrasound examination, clinicians can determine the nature and extent of lesions, including those that are difficult to localise during mammography exam (e.g. in the area of the thoracic wall). For this reason, ultrasound examination is considered to complement mammography. Therapeutic procedures, such as liquid cyst aspiration, abscess drainage, or biopsy for cytological or histologic study, can be performed under ultrasound guidance. Opinions vary regarding the use of particular biopsy techniques in specific clinical situations. Some authors prefer core needle biopsy [1, 2]; in other centres, fine-needle biopsy is equally popular $[3,4]$.

\section{Aim of the study}

The aim of the study was to redefine the role of fine-needle aspiration biopsy (FNAB) in the diagnosis of breast lesions, based on our own experience.

\section{Material and methods}

We conducted a retrospective analysis of the medical records of 680 patients who underwent breast ultrasound examination between April 2014 and December 2014, using an Aloka Alpha 6 with a linear probe at 7-12 $\mathrm{MHz}(\mathrm{Hi}-$ tachi, Wallingford, CT, USA). Some of them were qualified 
for biopsy according to current recommendations [5]. The indications for biopsy included the following:

- complex cysts (Breast Imaging - Reporting and Data System [BI-RADS] 4a),

- complex cysts with signs of inflammation (BI-RADS 3),

- benign solid lesions in the ultrasound image (BI-RDAS 3),

- lesions that might be neoplastic (BI-RADS 4),

- lesions that cannot be examined by systematic monitoring using imaging methods.

The biopsies were performed according to accepted standards $[5,6]$. The lesion was first localised, and then the puncture was performed under ultrasound guidance so that the needle tip was correctly placed within the pathologic structure of interest. Biopsy material was collected from the central part of the lesion, and, in the case of a lesion with central necrosis or fibrosis, from its edge. The aspirate was then distributed across the surface of a glass slide and fixed. Between two and four smears were prepared using the provided material. According to the guidelines, cytological results were classified as follows: C1: non-diagnostic; C2: benign; C3: suspicious, probably benign (atypical); C4: suspicious, probably malignant; and C5: malignant. [7]. Patients with suspicious smears were referred for core needle or surgical biopsy. Patients with benign cytological smears underwent repeated ultrasound examination at 6-month intervals during the following year.

The study was conducted under Institutional Review Board protocol no. KBUR D/2016/09/20 from 09.20.2016, University of Rzeszow. All experimental protocols were approved by the Institutional Ethics Committee. All methods were carried out in accordance with the approved guidelines.

\section{Statistical analysis}

The data were analysed using descriptive statistics, and results were reported as the mean, standard deviation, median (range of values), or number and percentage. The chi-square test was applied to test the significance of differences in the qualitative characteristics. Differences were considered statistically significant when $p<0.05$.

\section{Results}

In 359 of 680 cases, no pathologic structures were observed within the mammary glands. In 321 patients, the ultrasound examination revealed the presence of focal lesions. Among these patients, 107 qualified for biopsy. Patient age ranged from 20 to 64 years (mean and standard deviation are 40 and 10 years, respectively) in this sub-group. The abnormalities were diagnosed most frequently in the age groups 36-45 and 26-35 years (Fig. 1).
The ultrasound examination disclosed a slightly higher rate of lesions in the left breast; however, the differences were not statistically significant. Most of the abnormalities (60\%) were found in the upper-outer quadrant. The calculated $p$-value $(p=0.25)$ does not suggest a correlation between lesion location and individual breasts (left or right). The majority of lesions were smaller than $15 \mathrm{~mm}$. Abnormalities bigger than 15 $\mathrm{mm}$ accounted for $31 \%$ of all cases (Table 1 ).

All the obtained smears were diagnostic. Most of the results were categorised as benign lesions. Cancer cells were detected in six women. In one patient the lesion was classified as suspicious, probably malignant (C4). Detailed results are presented in Table 2.

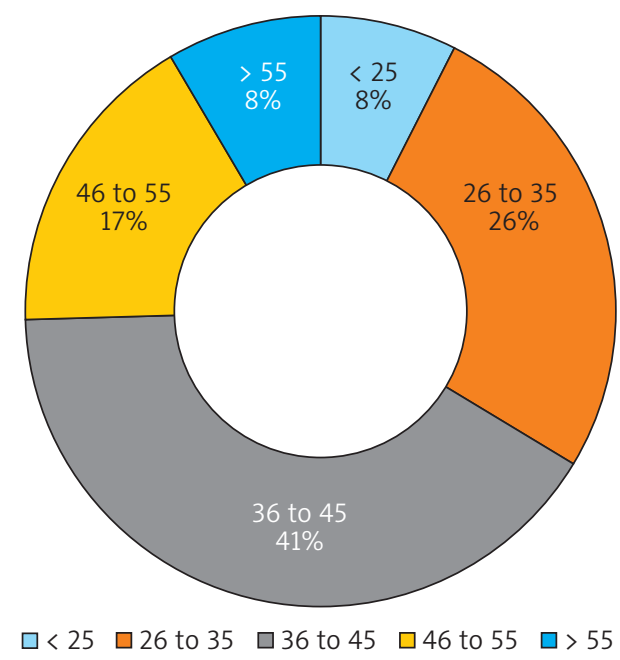

Fig. 1. Number of examined patients within different age groups

Table 1. Characteristics of lesions that qualified for fine-needle biopsy

\begin{tabular}{|c|c|c|c|}
\hline \multicolumn{3}{|c|}{ Breast lesions $(n=107)$} & \multirow{2}{*}{$\frac{p \text {-value }}{0.28}$} \\
\hline Diameter & $\leq 15 \mathrm{~mm}$ & $>15 \mathrm{~mm}$ & \\
\hline & $74(69 \%)$ & $33(31 \%)$ & \\
\hline Location & $\begin{array}{c}\text { Right } \\
(n=51)\end{array}$ & $\begin{array}{c}\text { Left } \\
(n=56)\end{array}$ & 0.25 \\
\hline Upper-outer quadrant & 31 & 33 & \\
\hline Lower-outer quadrant & 5 & 4 & \\
\hline Upper-inner quadrant & 12 & 9 & \\
\hline Lower-inner quadrant & 3 & 10 & \\
\hline
\end{tabular}

Table 2. Cytological findings among 107 aspirates

\begin{tabular}{lcc}
\hline Cytological diagnosis & Number & Percentage \\
\hline C1 Non-diagnostic & 0 & 0 \\
\hline C2 Benign & 99 & 92.5 \\
\hline C3 Suspicious, probably benign & 1 & 0.95 \\
\hline C4 Suspicious, probably malignant & 1 & 0.95 \\
\hline C5 Malignant & 6 & 5.6 \\
\hline
\end{tabular}


According to standard guidelines, open biopsy was performed in women with C4-C5 lesions [5]. In all cases, histopathological examination confirmed the presence of a malignant tumour. The patients were given appropriate oncological treatment.

In women with C2-C3 lesions, breast ultrasound examination was performed twice at 6-month intervals after the initial test. Follow-up tests showed no significant changes compared to the baseline examination, and none of the patients required extensive additional diagnostic examinations.

\section{Discussion}

Fine-needle aspiration biopsy is a diagnostic method that allows surgeons to determine the nature of breast lesions detected using imaging methods or clinical examination. According to Kocjan et al., FNAB plays an important role in:

- diagnosis of palpable nodular lesions that show no clinical signs of malignancy

- verification of breast cancer metastases

- preoperative staging of breast cancer, particularly when axillary lymph node metastasis is suspected [8].

To reliably perform guided biopsy, high-quality ultrasound equipment is necessary. According to the American College of Radiology, a high-frequency probe ( $\geq 10 \mathrm{MHz}$ ) is required [9]. The probe used in the present study complied with this guideline.

The primary objective of ultrasound examination is to identify a lesion, record its maximum size in two orthogonal sections on an image and in a written report, and identify the precise location of the lesion in the breast with respect to clock hours and distance from the nipple. Marking the orientation and location of the probe is also required. Recording these specific details during the course of the study allows for precise localisation of subtle focal lesions. Practice guidelines recommend against the excision of a detected lesion during the initial stage of diagnosis to determine whether it is benign or malignant. Instead, any type of biopsy is recommended, provided that the technique is reliable, repeatable, and safe. Fine-needle aspiration biopsy performed under ultrasound guidance meets all these requirements [4]. In addition, FNAB causes only slight discomfort, does not preclude activities of everyday life, eliminates scarring and organ deformation, and is not expensive.

Approximately $80 \%$ of biopsies reveal benign lesions. According to Cochrane et al., cancer is detected in only $6-12 \%$ of patients diagnosed in breast care centres [10]. Similar findings were revealed in the present study. The convergence of the cytological and histopathological findings of lesions scheduled for surgical excision and the lack of progression in patients undergoing follow-up ultrasound confirmed the accuracy and reliability of the procedure used.
Fine-needle aspiration biopsy has been criticised because it can yield false negative results. The sensitivity of biopsy to detect malignant lesions ranges from $85.6 \%$ to $97.1 \%$, and its specificity ranges from $83 \%$ to $100 \%$ [11-15]. For comparison, the values of these indicators for core needle biopsy are $85-100 \%$ and 86 $100 \%$, respectively [16]. According to Westenend et al., the positive predictive value of fine- and core needle biopsies is similar and ranges from $99 \%$ to $100 \%$ [17]. The only circumstance that justifies choosing core needle biopsy over fine-needle biopsy is the absence of an expert pathologist to evaluate cytological material [2].

FNAB has additional advantages over other available diagnostic methods (e.g. stereotactic biopsy, which is conducted under magnetic resonance guidance). FNAB enables comfortable supine positioning of the patient and eliminates the need for ionising radiation, which is an unavoidable element of the stereotactic biopsy, and allows for diagnosis in patients for whom magnetic resonance-guided biopsy is contraindicated.

Compared to core needle biopsy, FNAB is preferable in patients treated with anticoagulants, with lesions that are superficial or close to the chest wall, or in the vicinity of blood vessels or implants [16].

An important advantage of FNAB is the ability to obtain a definitive cytological diagnosis in 30-60 min [16, 18], which significantly reduces the patient's stress $[19,20]$.

FNAB is a safe procedure. Pain associated with the puncture is a major complication. Some reports describe the occurrence of pneumothorax during the puncture of lesions located at the chest wall, especially in the area of Spence's tail. The incidence of this complication is estimated to be $1 / 10,000[21,22]$. Haematomas at the injection site are usually small and are spontaneously resorbed, and those that require surgical intervention occur with a frequency of $<1 \%$ [21, 23-25]. Infection at the insertion site is even less common, and its frequency ranges from $0.01 \%$ to $0.2 \%$ [21, 24-26]. None of these complications occurred in our patients.

Diagnosing benign lesions during FNAB eliminates the need for open biopsy, provided that imaging and physical examination also indicate the benign character of the lesion [27-29]. Similarly, conclusive FNAB results reduce the need for surgical procedures in patients with malignant lesions [28, 30].

\section{Conclusions}

Based on the results of the present study, we offer two main conclusions that support the importance of FNAB in the diagnosis of breast lesions:

Fine-needle aspiration biopsy is a reliable method of assessing pathologic lesions in mammary glands.

Proper technique minimises the patient's discomfort during FNAB and helps to avoid serious complications. 


\section{Disclosure}

Authors report no conflict of interest.

\section{References}

1. Duijm LE, Groenewoud JH, Roumen RM, et al. A decade of breast cancer screening in The Netherlands: Trends in the preoperative diagnosis of breast cancer. Breast Cancer Res Treat 2007; 106: 113-119.

2. Candelaria RP, Hwang L, Bouchard RR, et al. Breast ultrasound: current concepts. Semin Ultrasound CT MR 2013; 34: 213-225.

3. Ajkay N, Bloomquist EV, Martin RC, et al. Fine-needle aspiration cytology in the evaluation of patients with radiographically occult, palpable breast abnormalities. Surgery 2015; 158: 946-952.

4. Takhellambam YS, Lourembam SS, Sapam OS, et al. Comparison of ultrasonography and fine needle aspiration cytology in the diagnosis of malignant breast lesions. J Clin Diagn Res 2013; 7: 2847-2850.

5. Stachura J, Niezabitowski A, Olszewski W, et al. Principles for the collection and analysis of thin-needle aspiration, thick-needle, and surgical bioptates, as well as material after radical breast surgery. Recommendations of the Polish Society of Pathologists, Polish Oncology Society, Polish Society of Surgeons, and Polish Society of Radiology. Med Prakt 2001; 7-8: 187-195.

6. Newell MS, Mahoney MC. Ultrasound-guided percutaneous breast biopsy. Tech Vasc Interv Radiol 2014; 17: 23-31.

7. Perry N, Broeders M, de Wolf C, et al. European guidelines for quality assurance in breast cancer screening and diagnosis. Fourth Editionsummary document. Ann Oncol 2008; 19: 614-622.

8. Kocjan G, Bourgain C, Fassina A, et al. The role of breast FNAC in diagnosis and clinical management: a survey of current practice. Cytopathology 2008; 19: 271-278.

9. ACR practice guideline for the performance of ultrasound-guided perutaneus brest interventional procedures" Revised 2014 (Resolution 7). Accesed December 20, 2014

10. Cochrane RA, Singhal H, Monypenny IJ, et al. Evaluation of genera practitioner referrals to a specialist breast clinic according to the UK national guidelines. Eur J Surg Oncol 1997; 23: 198-201.

11. Martelli G, Pilotti S, Coopmans de, et al. Diagnostic efficiency of physical examination, mammography, fine needle aspiration cytology (triple test) in solid breast lumps: an analysis of 1708 consecutive cases. Tumori 1990; 76: 476-479.

12. Reinikainen HT, Rissanen TJ, Piippo UK, et al. Contribution of ultrasonog raphy and fine-needle aspiration cytology to the differential diagnosis of palpable solid breast lesions. Acta Radiol 1999; 40: 383-389.

13. Ballo MS, Sneige N. Can core needle biopsy replace fine-needle aspiration cytology in the diagnosis of palpable breast carcinoma. A comparative study of 124 women. Cancer 1996; 78: 773 e7.

14. Barra Ade A, Gobbi H, de L Rezende CA, et al. A comparison of aspiration cytology and core needle biopsy according to tumor size of suspicious breast lesions. Diagn Cytopathol 2008; 36: $26 \mathrm{e} 31$.

15. Nagar S, lacco A, Riggs T, et al. An analysis of fine needle aspiration versus core needle biopsy in clinically palpable breast lesions: a report on the predictive values and a cost comparison. Am J Surg 2012; 204: 193-198.

16. Willems SM, van Deurzen CH, van Diest PJ. Diagnosis of breast lesions: fine-needle aspiration cytology or core needle biopsy? A review. J Clin Pathol 2012; 65: 287-292.

17. Westenend PJ, Sever AR, Beekman-De Volder HJ, et al. A comparison of aspiration cytology and core needle biopsy in the evaluation of breast lesions. Cancer 2001; 93: 146-150.

18. Kooistra B, Wauters C, Strobbe L, et al. Preoperative cytological and histological diagnosis of breast lesions: A critical review. Eur J Surg Oncol 2010; 36: 934-940.

19. Ubhi SS, Shaw P, Wright S. Anxiety in patients with symptomatic breast disease: effects of immediate versus delayed communication of results. Ann R Coll Surg Engl 1996; 78: 466-469.

20. Tan SM, Behranwala KA, Trott PA, et al. A retrospective study comparing the individual modalities of triple assessment in the pre-operative diagnosis of invasive lobular breast carcinoma. Eur J Surg Oncol 2002; 28: 203-208.
21. Meyer JE, Smith DN, Lester SC, et al. Large-core needle biopsy of nonpalpable breast lesions. JAMA 1999; 281: 1638-1641.

22. Bates T, Davidson T, Mansel RE. Litigation for pneumothorax as a complication of fine-needle aspiration of the breast. Br J Surg 2002; 89: 134-137.

23. Helvie MA, Ikeda DM, Adler DD. Localization and needle aspiration of breast lesions: complications in 370 cases. AJR Am J Roentgenol 1991; 157: 711-714.

24. Parker SH, Burbank F, Jackman RJ, et al. Percutaneous large-core breast biopsy: a multi-institutional study. Radiology 1994; 193: 359-364.

25. He Q, Fan X, Yuan T, et al. Eleven years of experience reveals that fineneedle aspiration cytology is still a useful method for preoperative diagnosis of breast carcinoma. Breast 2007; 16: 303-306.

26. Lipshy KA, Neifeld JP, Boyle RM, et al. Complications of mastectomy and their relationship to biopsy technique. Ann Surg Oncol 1996; 3: 290-294.

27. Green B, Dowley A, Turnbull LS, Smith PA, et al. Impact of fine-needle aspiration cytology, ultrasonography and mammography on open biopsy rate in patients with benign breast disease. Br J Surg 1995; 82: 1509-1511.

28. Rubin M, Horiuchi K, Joy N, et al. Use of fine needle aspiration for solid breast lesions is accurate and cost-effective. Am J Surg 1997; 174: 694696.

29. Morris KT, Pommier RF, Morris A, et al. Usefulness of the triple test score for palpable breast masses. Arch Surg 2001; 136: 1008-1012.

30. White RR, Halperin TJ, Olson JA Jr, et al. Impact of core-needle breast biopsy on the surgical management of mammographic abnormalities. Ann Surg 2001; 233: 769-777. 